\title{
Debates atuais em humanização e saúde: quem somos nós?
}

\author{
Current debates on humanization and health: who are we?
}

Rosângela M inardi M itre Cotta ${ }^{1}$

Roberta Sena Reis ${ }^{1}$

AlineA parecida de Oliveira Campos ${ }^{1}$

Andréia Patrícia Gomes ${ }^{1}$

Vanderson Espiridião Antonio ${ }^{1}$

Rodrigo Siqueira-Batista ${ }^{1}$

${ }^{1}$ Departamento de Nutrição eSaúde, Universidade Federal deViçosa. Avenida P. H. Rolfs s/n, Campus Universitário. 36571-000 Viçosa M G.

rmmitre@ufv.br
Abstract Bearing in mind that the Brazilian Unified Health System (SUS) is a social process in construction, and as health professionals are important individuals in this process, the role of permanent education as an important instrument to ensurehumanized careishighlighted. Thescope of this paper is to discuss the experience of the training course for health professionals of a public health outpatient unit, based on the prospect of humanized treatment, seeking the implementation of a sanitary model committed to the formal values contained in the SU S ideals. Theteaching-learning methodology used is based on problem-solving, derived from the processing of a problem situation taken from the experience of teachers. The professi onals identified that the standard established in the way of thinking and acting in health is unsatisfactory to meet the challenges faced in the sector. The strategies used contributed to systematize the content through reflection on the theoretical benchmarks submitted, by eliciting reflective and critical thought, which are fundamental aspects for broadening and increasing the process of empowerment of professionals. The course stimulated group action, placing the discussion on the humanization of health actions on the agenda.

Key words Humanization in health, Active education methodology, Learning, Ongoing education
Resumo Introdução: Considerando ser o SU S um processo social em construção, e sendo os profissionais de saúde importantes sujeitos desse processo, destaca-se o papel da educação permanente como um relevante instrumento para a garantia do cuidado humanizado. Objetivo: discutir a experiência do curso de capacitação dos profissionais de saúde de uma U nidade de Saúde públicaambulatorial, com base na perspectiva da humanização, visando a implementação de um modelo sanitário comprometido com os valores essenciais impressos nos ideais do SUS. M étodos: A me todologia de ensino-aprendizagem utilizada baseou-sena problematização, utilizando-se do processamento de uma situação problema elaborada a partir da experiência dos docentes. Resultado: Os profissionais identificaram que o padrão instituído no modo de pensar e fazer em saúde éinsati sfatório para suprir os desafios enfrentados no setor. As estratégias utilizadas contribuíram para sistematizar o conteúdo através da reflexão sobre os referenciais teóricos apresentados, ao estimular o pensamento reflexivo e crítico, aspectos estes fundamentais para ampliar e aprofundar o processo de empoderamento dos profissionais. Conclusão: o curso estimulou a grupalidade, colocando em pauta na agenda, a discussão sobre a humanização das ações em saúde. Palavras-chave Humanização em saúde, M etodologia ativa de ensino aprendizagem, Educação permanente 
Introdução

O movimento da Reforma Sanitária representou uma mudança paradigmática na saúde do Brasil não apenas pela ênfase dada à Atenção Primária à Saúde (APS), mas também pela importância dos determinantes sociais no processo saúde doença $\mathrm{a}^{1-3}$.

Com a criação do Sistema Único de Saúde (SUS) a saúde passa a ser direito de todo cidadão brasileiro, sendo dever do Estado prover políticas econômicas esociais que visem a redução dos ris$\cos$ de adoecer ${ }^{4}$. Especificamente, relacionado às ações e serviços, estas devem ser garantidas por meio de políticas de promoção, proteção e recuperação, com participação da sociedade civil organizada nas decisões e na implementação das ações ${ }^{3,5-8}$.

Na trajetória de construção do SUS, há que se destacar os muitos avanços obtidos, especialmente aqueles relacionados à universalidade e à descentralização. Não obstante, ainda persistem alguns entraves, apontando para a necessidade de aperfeiçoamento na implementação do sistema em suas diretrizes essenciais, tais como a re gionalização e a hierarquização, a equidade, a integralidade, uma efetiva participação social eo investimento na formação e capacitação de re cursos humanos em saúde 3,8-10.

Dentre os principais obstáculos presentes no cotidiano do SUS, salienta-se a presença marcante do modelo biomédico na práxis dos profissionais de saúde ${ }^{2,711}$, as relações de trabalho precarizadas, a fragilidade na relação usuárioserviço de saúde, a necessidade de ampliação do acesso com qualidade aos serviços de saúde, a pobre participação dos usuários na gestão dos serviços, o despreparo profissional aliado ao modelo centralizado e vertical ${ }^{10}$, ou seja: a implantação e execução das políticas públicas no país ainda não éexercida demodo satisfatório $0^{7,12}$.

Frente à necessidade de mudança do cenário vigente, foi instituído a "Política Nacional de Humanização da Atenção e Gestão no Sistema Ú nico deSaúde", entendida como uma estratégia de fortalecimento do sistema público de saúde. Seu propósito é o de contribuir para a melhoria da qualidade da atenção e da gestão da saúde no Brasil, por meio do fortalecimento da humanização como política transversal na rede, afirmando a indissociabilidade do modelo de atenção e de gestão ${ }^{10}$. N estes termos, as principais prioridades al vo da Política Nacional de H umanização (PNH) são: construção de autonomia e protagonismo dos sujeitos e coletivos implicados na rede do SU S; corresponsabilidadedesses sujeitos nos processos de gestão e aten ção; fortal ecimento do controle social com caráter participativo em todas as instâncias gestoras do SUS; fortalecimento de trabalho em equipe multiprofissional, fomentando a transversalidade e a grupalidade; apoi o à construção de redes cooperativas, solidárias e comprome tidas com a produção de saúde edesujeitos; valorização da dimensão subjetiva e social em todas as práticas de atenção e gestão no SUS, fortal ecendo o compromisso com os direitos do cidadão, destacando-se o respeito às questões de gênero, etnia, orientação sexual e às populações específicas ${ }^{10}$.

Por humanização entende-se "a valorização dos diferentes sujeitos implicados no processo de produção de saúde: usuários, trabalhadores e gestores" ${ }^{\prime 10}$. 0 protagonismo dos sujeitos e sua autonomia, o estabelecimento de vínculos solidários entre os atores, a participação coletiva no processo de gestão, bem como a elaboração de tramas de cooperação norteiam essa política ${ }^{10}$.

A humanização como estratégia de intervenção nos processos de trabalho e na geração de qualidade da saúde, vai ao encontro dos princípios do SUS, ao enfatizar a necessidade de se assegurar atenção integral à população, bem como estratégias que ampliem a condição de direitos e de cidadania dosindivíduos. A humanização de pendeainda de modificações no modo de pensar e agir das pessoas, na ênfase aos valores atrelados à defesa da vida, a fim de tornar criativo e prazeroso o modo de fazer o trabalh ${ }^{13,14}$.

Considerando ser o SUS um processo social em construção, e sendo os profissionais de saúde importantes sujeitos desse processo, destaca-se o papel da educação permanente como um dosmais relevantes instrumentos para a garantia de um cuidado humanizado. Assim, o objetivo destetrabalho foi discutir a experiência do curso de capacitação dos profissionais de uma Unidade deSaúde, com base na perspectiva da humanização, visando a sensibilização para o exercício pleno e conscientedeseu papel na implementação deum modelo de sistema sanitário comprometido com os valores essenciais dealteridadee solidariedade, impressos nos ideais do SUS.

\section{Métodos}

Em outubro de 2009, realizou-se, o curso de capacitação intitulado “D ebates A tuais em Humanização e Saúde: Quem Somos Nós?", por de manda dos próprios profissionais da Unidade de Saúde pública ambulatorial, da Universidade 
Federal deViçosa (USU FV), instituição esta, que atende aos respectivos docentes, discentes, pessoal técnico-administrativo e familiares.

As atividades desenvolvidas no curso tiveram como objetivo central aperfeiçoar os conhecimentos referentes à $\mathrm{PNH}$, visando melhoria do cuidado oferecido ao público alvo, assim como do espaço de trabalho (social, afetivo, físico e técnico) dos profissionais da USUFV. O curso teve duração de 16 horas, sendo o mesmo realizado a partir de exposições dialogadas, seguidas de dinâmicas de grupo e processamento de uma Situação Problema (SP). Os temas abordados durante a realização do curso foram: Humanização no Sistema Único deSaúde; Bioética ehumanização; Mudança na formação e no trabalho em saúde; humanização e o trabalho em saúde: 0 atendimento na sala de trauma, na urgência e emergência.

A seguir, apresentamos a SP trabalhada ao longo do curso.

Um dia na Unidade de Atenção Primária à Saúde (UAPS) de Saramandaia

M aria Alicetrabalha como médica em uma UAPS, desde 2005. Estudou muito durante sua formação, sendo aluna dedicada do começo ao fim do curso. Contudo, quando se formou e foi trabalhar como médica, tomou consciência de que não conseguia, em muitas oportunidades, juntar coerentemente os saberes necessários para resolver os problemas dos usuários que a procuravam precisando de ajuda. Tinha ouvido falar do Sistema Ú nico de Saúde do Brasil, o SUS, dito um dos mais avançados do mundo, que garantia o acesso universal, a integralidade do cuidado, mas não sabia di reito como garantir que seus pacientes fossem atendidos nasunidades secundárias, quando os mesmos precisavam de um especialista. Tinha ouvido falar de escuta ativa, de clínica ampliada, mas via a agenda sempre cheia, a equipe super estressada, os colegas mal olhando para os pacientes...

Conversando com a enfermeira Zildete escutava as mesmas queixas, os mesmos questionamentos. Em sua formação, na escola de enfermagem, ninguém havia falado das filas, da equipe sobre carregada, do número enorme de famílias excedente. Como fazer as visitas domiciliares, supervisionar as agentes comunitárias e ainda preencher a papelada com o tempo que tinha? H umanização? Ética? Bi oética? 0 jeito era correr para atender as pessoas que já estavam reclamando, pois tinham chegado à unidade às seis horas... ejá eram dez horas da manhã..
- Entra o próximo, chamou a Dra. M aria Alice.

- Bom dia doutora. Vim colher o preventivo, disse a paciente.

- Qual o seu nome?

- Ana Maria.

- Ana, acabou a lâmina. Você tem que voltar semana que vem... M aria Alice falou sem olhar para a paciente.

- M as estou esperando desde as seis horas...

- M inha filha, não posso fazer nada! A culpa é do governo que não mandou o material!

- M as tem dois anos que não faço o exame... E semana que vem preciso ir trabalhar...

- Volta semana que vem... Já disse... Ah! A proveita ali no guichê e mande a próxima entrar!

E assim, transcorre mais um dia de trabalho na UAPS

Os profissionais foram incentivados a participar como agentes de transformação social na busca de soluções originais vinculadas a prática cotidiana do trabalho ${ }^{15,16}$.

Para a realização das dinâmicas os participantes foram divididos em três grupos, privilegiando a heterogeneidade profissional. Foram utilizados como materiais de apoio: papel cartão em tamanho de cartolina, pincéis atômicos, foIhas de papel ofício, canetas, entre outros. Cada grupo foi acompanhado por um tutor, queatuou como facilitador da discussão, incentivando a integração e a participação de todos.

A problematização realizada nas dinâmicas permitiu que os partici pantes tomassem contato com as informações e a produção do conhecimento, promovendo assim, o seu próprio desenvolvimento ${ }^{17,18}$. Esta forma de aprendizagem representou um instrumento necessário e significativo para ampliar as possibilidades, as reflexões e os caminhos, permitindo o exercício da liberdade e da autonomia na realização de escoIhas e na tomada de decisões ${ }^{18,19}$.

A SP foi distribuída aos participantes, que divididos em pequenos grupos, discutiram as questões abordadas e formularam duas questões de aprendizagem, a saber: Como transformar a realidade da U nidade de A tenção Primaria a Saúde(UAPS) no ideal formulado para o SUS?, e Como a formação do profissional de saúde pode transformar a realidade?

As discussões em pequenos grupos possibilitaram a construção coletiva de cartazes, instrumento eleito por se tratar deum recurso simples e eficiente para despertar o interesse e curiosidade dos participantes ${ }^{20}$. Posteriormente, cada grupo apresentou para o grande grupo suas sínteses coletivas, permitindo assim, um amplo debate 
coletivo em roda de conversa e configuração de uma nova síntese, neste momento com todos os participantes.

\section{ResultadoseDiscussão}

Participaram do curso 23 profissionais de dife rentes categorias - médicos, dentistas, enfermeiros, auxiliares de enfermagem, fisioterapeutas, auxiliares de laboratórios e pessoal técnico-administrativo.

As Figuras 1, 2 e o Quadro 1 apresentam os cartazes confeccionados pelos profissionais de saúde da USUFV em relação aos pontos discutidos na resolução das questões de aprendizagem propostas. A partir dos materiais elaborados pelos participantes, iniciou-se um processo de reflexão-análise das questões levantadas pelo grupo. As atividades realizadas entre os participantes permitiram o debate do cotidiano de trabal ho na USU FV, os impasses vivenciados no dia a dia e a criação de estratégias coletivas para a superação dessas dificuldades.

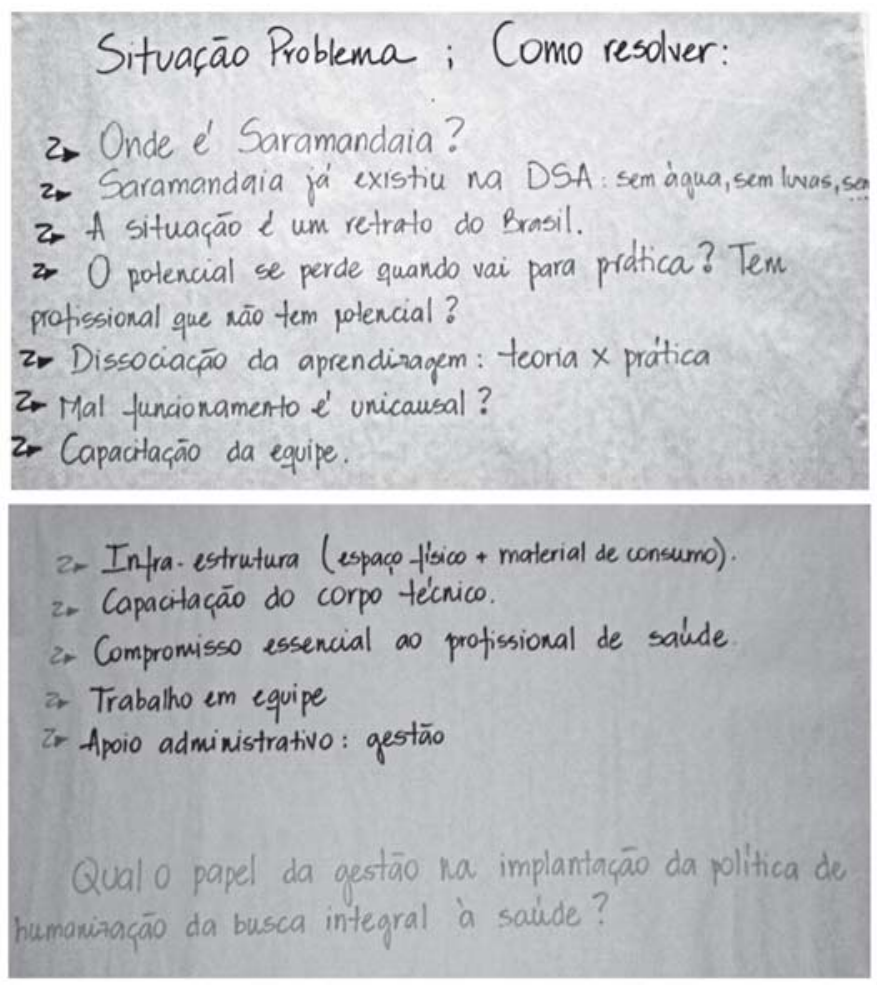

Figura 1. Processamento da Situação Problema do Grupo 1.
Na Figura 1 evidencia-se o papel do Gestor de Saúde na implantação da política de humanização, relatado pelos profissionais após reflexãoanálise das questões levantadas pelo grupo, se guido da construção da pergunta Q ual o papel da gestão na implantação da política de humanização da busca integral à saúde?

A dificuldade degestão observada na SP, aliada à desvalorização do trabalho e do trabalhador, tanto por parte dos gestores municipais quanto pelo gerenteda UAPS, apontam para uma realidade comumente observada em diversos municípios do país. Segundo dados do M inisté rio da Saúde, a gestão dos recursos humanos é apontada como uma das dificuldades para implantação adequada do SUS desde a sua criação ${ }^{21}$. M oreira e Pelizzaro ${ }^{22}$ afirmam que a visão desmedicalizada da saúde, debatida desde muito pelo M ovimento da Reforma Sanitária, impulsiona a busca constante por superação do trabaIho fragmentado e unidimensional em saúde.

Aspectos referentes à formação profissional vêm ganhando contornos próprios no trabalho em saúde, na medida em que a indissociabilidade entre teoria e prática, o desenvolvimento de uma visão integral do homem e a ampliação da concepção de cuidado tornam-se prementes para 0 adequado desempenho laboral ${ }^{17}$. A dificuldade em seencontrar profissionais com formação acadêmica voltada para o paradigma da Produção Social da Saúde, nos remete a repensar o modo de atuação destes profissionais frente à saúde no paí $5^{23}$. Gomes et al. ${ }^{24}$ ressaltam a importância do processo de aprendizagem que permita aos estudantes a aquisição de conhecimentos teóricos, com base na observação da realidade e na reflexão crítica sobre as ações dos sujeitos, de forma queos conteúdos teóricos sejam apreendidos em conexão com a prática.

Relatos como o potencial se perde quando se vai a prática e dissociação entre teoria e prática (Figura 1) corroboram com os achados de Stella et al. ${ }^{25}$, uma vez que, embora as escolas possibilitem que os estudantes se insiram em cenários de práticas profissionais, estas apontam para carências que precisam da formulação de novas políticas públicas que, implementadas, sejam capazes de impulsionar e sustentar as mudanças.

Segundo Salgueiro et al. ${ }^{26}$, o governo tem se empenhado na elaboração de políticas públicas capazes de oferecer maior ressonância eviabilidade na busca da qualidade e humanização em saúde.

Outro ponto destacado pelos participantes do curso de capacitação foi anecessidade deequipes e técnicos capacitados para atender às neces- 
sidades da população (Figura 1), sendo que 0 fundamental é perceber que o importante para a humanização, é justamente a permeabilidade do técnico ao não técnico, o diálogo entre essas dimensões interligada ${ }^{27}$.

Guedes et al. ${ }^{28}$ salientam que as equipes de saúde, muitas vezes, têm dificuldade para dialogar e problematizar situações inerentes ao cotidiano, uma vez que se privilegiam os processos de trabalho em si, em detrimento dos sujeitos dotados de conhecimentos e histórias vivenciadas. Gomes et al. ${ }^{24}$ afirmam que os processos de capacitação dos trabalhadores devem tomar como referência as necessi dades de saúde da população, da gestão e do controle social para qualificar as práticas de saúde e a educação dos profissionais e melhorar a atenção à saúde. 0 procedimento educativo contextualizado no processo detrabal ho agrega o saber científico àquele que emerge do campo para potencializar o conhecimento a partir de ações técnicas e políticas realizadas pelos trabalhadores ${ }^{29}$.

A qualidade dos serviços de saúde resulta de diferentes fatores que se culminam em instrumentos, o que contribui tanto para a definição e análise de possíveis problemas, como para a avaliação do empenho dos profissionais de saúde com as normas técnicas, sociais e humanas ${ }^{30}$.

A formação acadêmica fragmentada e a discrepância entre o aprendido no meio acadêmico e o real vivenciado pelo profissional de saúde foram pontos citados pelos participantes do curso (Quadro 1), aspectos que contribuem para que

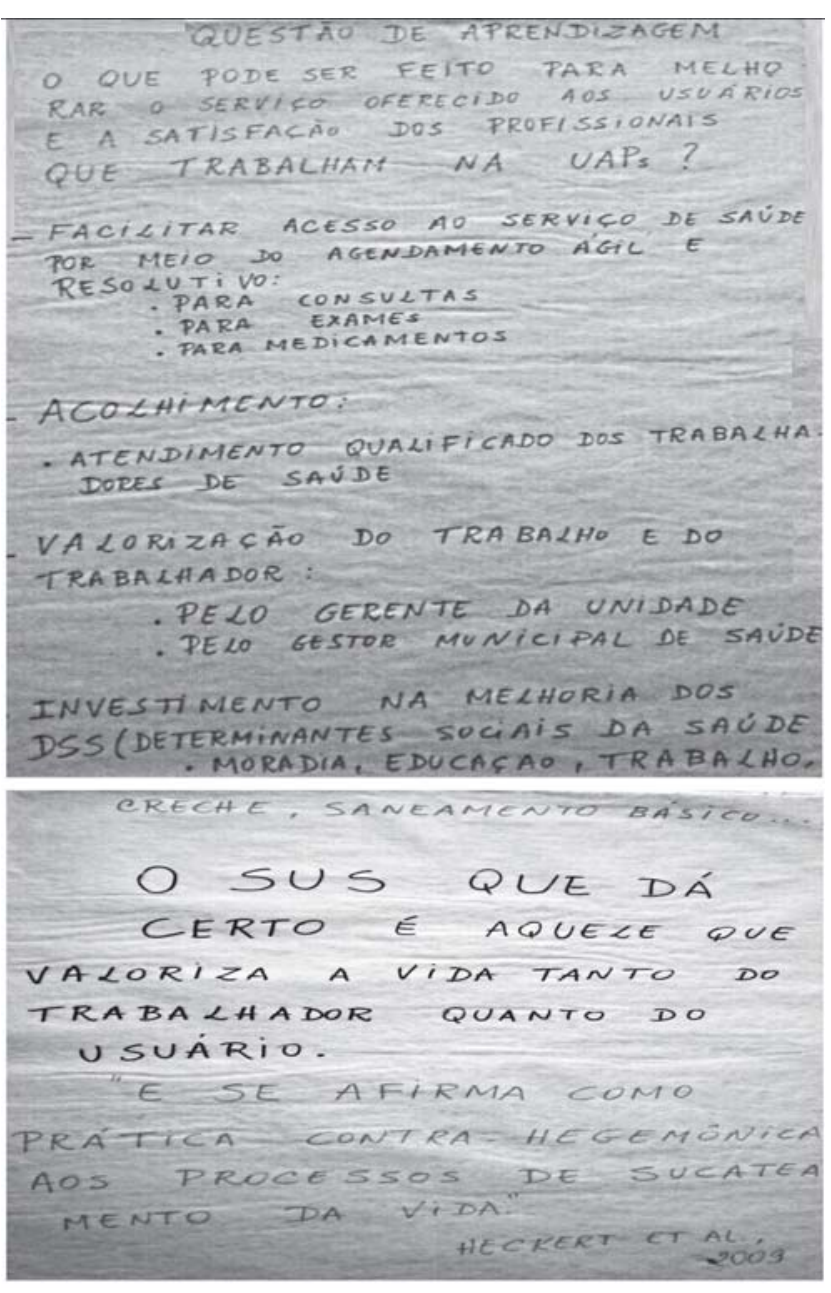

Figura 2. Processamento da Situação Problema do Grupo 3.

Quadro 1. Processamento da Situação Problema do Grupo 2

\begin{tabular}{|l|}
\hline TEM AS DISCUTID OS \\
\hline Unidade de Atenção Primaria a Saúde (UAPS) \\
Escuta ativa \\
Clínica ampliada
\end{tabular}


o profissional de saúde não saiba lidar com os entraves enfrentados.

Ainda hoje, a educação em saúde tem sido fundamentada, tradicionalmente, em metodologias de transmissão de conhecimentos, sem se levar em consideração que, o cenário atual ne cessita de profissionais formados com perfil crítico-reflexivo e com habilidades para trabalho em equipes ${ }^{18,24,31}$. Torna-se necessário, portanto, ajustar o perfil do profissional de saúde às ações desenvolvidas pelo SUS, através da parceria das instituições formadoras com os serviços de saú$\mathrm{de}^{32}$. Tais mudanças devem ser orientadas de acordo com as necessidades de saúde da população. D efender uma formação profissional voltada para atuar no SUS implica concebê-lo como um importante projeto político em defesa da saúde pública ${ }^{18,24}$.

Segundo Ferreira et al. ${ }^{33}$, o modo através do qual cada profissional de saúde enxerga o mundo, acaba por determinar um estilo de pensamento, o qual adota como verdade absoluta. A partir de um conhecimento clínico, muitos profissionais se preocupam apenas em tratar o paciente, enão em cuidá-lo.

Desde esta perspectiva, como diretriz essencial da humanização destaca-se 0 acolhimento. 0 acolhimento ao usuário foi outro ponto citado pelos profissionais de saúde da USU FV. As fal has no acolhimento por parte da equipe e da gestão (Quadro 1 e Figura 2) mostram os entraves e percalços ainda existentes no setor saúde. A postura de acolhimento da equipe de saúde se revela através de atitudes tais como: concentração no atendimento, aproximação ao clienteesentimento em relação aos seus problemas ${ }^{34}$. 0 acolhimento à pessoa que busca o cuidado em saúde se manifesta na relação ética que se estabelece entre o usuário eo profissional, ou, em outros termos, entre aquele que é cuidado e aquele que cuida. A garantia de acesso ao serviço de saúde para os usuários representa a responsabilidade do serviço para com suas necessidades de saúde ${ }^{35,36}$.

0 acolhimento otimiza 0 acesso dos usuários aos serviços de saúde, humanizando as relações entre os profissionais e aqueles que buscam por atenção sanitária, referindo-se à forma de recepcionar os usuários e de escutar seus problemas e/ou demandas, em uma abordagem que contemplenão apenas a dimensão biológica, mas também a psicológica, a social, a cultural e a ambiental, dentre outras possíveis $s^{36,37}$.

Para que as necessidades dos usuários sejam satisfeitas demaneira integral éfundamental que a atividade de recepção do usuário no serviço ocorra de maneira acolhedora ${ }^{38}$. N estes termos, o acolhimento aos usuários do SUS deve se dar segundo a necessidade de cada um. É relevante a presença de uma equipe humanizada que atue como facilitadora na concretização de estratégias de acol himento para que o acesso aos serviços de saúde contemple o princípio da integralida$\mathrm{de}^{34}$. 0 acolhimento, enquanto estratégia para reconfigurar o processo de trabalho, otimiza 0 acesso dos usuários aos serviços de saúde, humanizando as relações entre o profissional de saúde e o paciente, contemplando as dimensões biológicas, psicológica, social ecultural ${ }^{37}$.

Outro aspecto merecedor de destaque refere se ao não ol har nos ol hos do paciente, observado e relatado pelos profissionais de saúde da USUFV (Q uadro 1). Segundo Deslandes eM itre ${ }^{39}$, o processo comunicativo, em seu cotidiano, marcado muitas vezes pelos encontros e desencontros dos diferentes atores envolvidosno setor saúde, ocorre no contexto das relações estabelecidas pelas desigualdades, ainda vigente, entre seus pacientes e profissionais de saúde. Igualmente, a escuta, o acolhimento, a negociação, a interpretação de histórias e a aprendizagem com a experiência do outro constituem-se em pontos cruciais para o desenvolvimento de um serviço humanizado no setor saúde ${ }^{40}$.

\section{Considerações Finais}

A metodologia ativa de ensino-aprendizagem e neste contexto, a problematização, destaca-se como estratégica inovadora, por estimular a discussão e a análise crítica dos temas apresentados, fundamentais para 0 aprendizado ${ }^{41}$ configurando, portanto, uma das possibilidades de discutir o tema na instituição. Tal estratégia afirma as redes de serviços como espaços de sociabilidade, de trocas, em que se enfatiza a produção de saúde como produção de subjetividades, colocando em questão práticas disciplinares ${ }^{28}$. Assim, as estratégias utilizadas contribuíram para sistematizar os conteúdos trabalhados, através da reflexão sobre os referenciais teóricos apresentados, estimulando o pensamento reflexivo e crítico, aspectos estes fundamentais para ampliar e aprofundar o processo de empoderamento dos profissionais de saúde da USUFV e conse quentementea consolidação das políticas de saúde, em especial a PNH.

0 processo vivenciado no curso foi marcado por discussões e (re) construções queiam se formando ao longo do desenvolvimento das ativi- 
dades. Destarte, resgatar o sentido da prática profissional em saúde, bem como reconhecer a importância de se trabalhar nestas organizações, através da reflexão e análise dos atores envolvidos, poderá contribuir para implementar transformações no processo de trabalho via humanização dos serviços.

Por fim, conclui-se que o curso estimulou a grupalidade, colocando em pauta na agenda da USUFV, a discussão sobre a humanização das ações em saúde.

\section{Colaboradores}

RM M Cotta foi responsável pela idealização, delineamento do objeto de estudo, desenho da metodologia, coleta e análise dos dados, redação e revisão do manuscrito do manuscrito. RS Reis, AAO Campos, AP Gomes eVE Antonio colaboraram com o processamento dos dados e auxiliaram na redação do manuscrito e revisão critica final. RS Batista foi responsável pela coordenação, planejamento, orientação metodológica, acompanhamento do trabalho, redação e revisão do manuscrito. 


\section{Referências}

1. Cohn A. A reforma sanitária brasileira após 20 anos do SUS: reflexões. Cad Saude Publica 2009; 25(7): 1614-1619.

2. Gomes KO, Cotta RM M, Euclydes MP, Targueta $\mathrm{CL}$, Priore SE, Franceschini SCC. Avaliação do impacto do Programa Saúde da Família no perfil epidemiológico da população rural de Airões, M unicípio de Paula Cândido M G (1992-2003). Cien Saude Colet 2009; 14(Supl.1):1473-1482.

3. Martins PC, Cotta RM M, Siqueira-Batista R, M endes FF, Franceschinni SCC, Priore SE, Dias, G. Democracia e empoderamento no contexto da promoção da saúde: possibilidades e desafios apresentados ao Programa de Saúde da Família. Physis 2009; 19(3):679-694.

4. Cotta RM M, M endes FF, Muniz JN. Descentralização das políticas públicas de saúde: do imaginário ao real. Viçosa: UFV, Cebes; 1998.

5. Backes DS, Koerich MS, Rodrigues ACRL, Drago LC, Klock P, Erdmann AL. O que os usuários pensam e falam do Sistema Ú nico de Saúde? U ma análise dos significados à luz da carta dos direitos dos usuários. Cien Saude Colet 2009; 14(3):903-1010.

6. Cotta RM M, Cazal M M, Rodrigues JFC. Participação, Controle Social e Exercício da Cidadania: a (des)informação como obstáculo à atuação dos conselheiros de saúde. Physis 2009; 19(2):419-438.

7. Costa GD, Cotta RMM , Ferreira M LSM, Reis JR, Franceschini SCC. Saúde da Família: desafios no processo de reorientação do modelo assistencial. Rev Bras Enferm 2009; 62(1):113-118.

8. Martins PC, Cotta RM M, M endes FF, Reis JR, Franceschini SCC. Conselhos de Saúde e a Participação Social no Brasil:Matizes da Utopia. Physis 2008; 18(1):105-121.

9. Siqueira-Batista R, Schramm FR. A saúde entre a iniqüidade e a justiça: contribuições da igualdade complexa de Amartya Sen. Cien Saude Colet 2005; 10(1):129-142.

10. Brasil. M inistério da Saúde (MS). Secretaria de Atenção à Saúde. Núcleo Técnico da Política de Humanização. Humaniza SUS: documento base para gestores e trabalhadores do SU S. 4a Edição. Braślia: MS; 2010.

11. Ferreira MLS, Cotta RMM, Oliveira MS. Reconstrução Teórica do Cuidado para as Práticas de Saúde: Um Olhar a Partir da Produção de Alunos de Curso de Especialização a Distancia. Rev Bras Educ M ed 2008; 32(3):291-300.

12. Pinheiro R. Democracia e saúde: sociedade civil, cidadania e cultura política. Physis 2004; 4(1):1114.

13. Campos GWS. Humanização da saúde:um projeto em defesa da vida? Interface Comun Saúde Educ 2005; 9(17):389-406.

14. Santos-Filho SB. Perspectivas da avaliação na Política Nacional de Humanização em Saúde: aspectos conceituais e metodológicos. Cien Saude Colet 2007; $12(4): 999-1010$
15. Bordenave JED. Alguns fatores pedagógicos. In Santana JP, Castro JL, organizadores. Capacitação em Desenvolvimento de Recursos Humanos - CADRHU. Natal: MS, OPAS; 1999. p. 261-268.

16. Bordenave J, Pereira A. Estratégias de ensino-aprendizagem. 26a Edição. Petrópolis: Vozes; 2004.

17. M itre SM, Siqueira-Batista R, Girardi-M endonça JM G, M orais-Pinto N M, M eirelles CAB, Pinto-Porto C, M oreira T, Hoffmann LMA. M etodologias ativas de ensino-aprendizagem na formação profissional em saúde: debates atuais. Cien Saude Colet 2008; 13(Supl. 2):2133-2144.

18. Cotta RM M, Silva LS, Lopes LL, Gomes KO, Cotta FM, Lugarinho R, Mitre SM M. Construção de portfólios coletivos em curriculos tradicionais: uma proposta inovadora de ensino-aprendizagem. Cien Saude Colet 2010; 17(3):787-796.

19. Cyrino EG, Toralles-Pereira M L. Trabalhando com estratégias de ensino-aprendizado por descoberta na área da saúde: a problematização e a aprendizagem baseada em problemas. Cad Saude Publica 2004 20(3):780-788.

20. Turano W, Almeida CCC. M étodos e técnicas aplicados à educação nutricional. In: Gouveia ELC, organizadores. Nutrição, Saúde e Comunidade. Rio de Janeiro: Revinter; 1999. p. 107-135.

21. Brasil. M inistério da Saúde (MS). Secretaria de Políticas de Saúde. Departamento de Atenção Básica. Cadernos de Atenção Básica: Programa Saúde da Família. Brasília: M S; 2000.

22. Moreira B, Pellizzaro I. Educação em Saúde: um programa de extensão universitária. Rev Textos \& Contextos 2009; 8(1):156-171.

23. Vilela EM, M endes IJM. Interdisciplinaridade e saúde: estudo bibliográfico. Rev Lat Am Enfermagem 2003; 11(4):525-531.

24. Gomes MPCG, Ribeiro VMB, M onteiro DM, Leher EMT, Louzada RCR. O uso de metodologias ativas no ensino de graduação nas ciências sociais e da saúde - avaliação dos estudantes. Cien Educ 2010; 16(1):181-198.

25. Stella RCR, Abdalla IG, Lampert JB, Perim GL, Silva RHA, Costa NM SC. Cenários de Prática e a Formação M édica na Assistência em Saúde. Rev Bras Educ M ed 2009; 33(Supl.1):63-69.

26. Salgueiro JB, Ramos MZ, Falk M LR, Raymundo M M , Schenkel SS. Avaliação das ações humanizadoras desenvolvidas na pediatria do Hospital de Clínicas de Porto Alegre. Rev Hosp Clin Porto Alegre 2007; 27(2):5-9.

27. Ayres JRC. O cuidado, os modos de ser (do) humano e as práticas de saúde. Saude Soc 2004; 13(3):1629.

28. Guedes $C R$, Pitombo L, Barros MEB. Os processos de formação na Política Nacional de Humanização: a experiência de um curso para gestores e trabalhadores da atenção básica em saúde. Physis 2009; 19(4):1087-1109. 
29. Silva JAM, Ogata M N, Machado M LT. Capacitação dos trabalhadores de saúde na atenção básica: impactos e perspectivas. Rev Eletr Enfer [periódico na Internet]. 2007 maio-ago [acessado 2010 jun 26]; 9(2):[cerca de 13 p.]. Disponível em: http://www. fen.ufg.br/revista/v9/n2/v9n2a08.htm

30. Cotta RM M, Azeredo CM, Schott M, M artins PC, Franceschini SCC, Priore SE. Sobre o conhecimento e a consciência sanitária brasileira: o papel estratégico dos profissionais e usuários no sistema sanitário. Rev M ed M inas Gerais 2006; 16(1):2-8.

31. Cotta RM M, Gomes AP, M aia TM, Magalhães KA, $M$ arques ES, Siqueira-Batista R. Pobreza, injustiça e desigualdade social: repensando a formação de profissionais de saúde. Rev Bras Educ M ed 2007; 31(3):278-286.

32. Rios IC. Humanidades e medicina: razão e sensibilidade na formação médica Cien Saude Colet 2010; 15(Supl. 1):1725-1732.

33. Ferreira M LSM, Cotta RM M , Oliveira MS. Construção Coletiva de Experiências Inovadoras no Processo Ensino-aprendizagem na Formação de Profissionais da Saúde. Rev Bras Educ M ed 2009; 33(2): 240-246.

34. Souza ECF, Vilar RLA, Rocha NSPD, Uchoa AC, Rocha PM. Acesso e acolhimento na atenção básica: uma análise da percepção dos usuários e profissionais de saúde. Cad Saude Publica 2008; 24(Supl. 1): $100-110$.

35. Coelho M O, Jorge MS. Tecnologia das relações como dispositivo do atendimento humanizado na atenção básica à saúde na perspectiva do acesso, do acolhimento e do vínculo. Cien Saude Colet 2009; 14(Supl.1):1523-1531.

36. Siqueira-Batista R. 0 cuidado integral em questão: diálogos entre filosofia e medicina. Rev Bras M ed 2010; 47(3):273-275.

37. Moraes SDTA, Buchalla CM, Valenti VE, Leite $L$, Tanaka ACD, Macedo Junior H, Abreu LC. AcoIhendo 0 acolhedor: 0 caminho mais curto para a humanização da assistência. Rev Bras Cres Desenv Hum 2009; 19(3):393-402.

38. Takemoto MLS, Silva EM. Acolhimento e transformações no processo de trabalho de enfermagem em unidades básicas de saúde de Campinas. Cad Saude Publica 2007; 23(2):331-340.

39. Deslandes SF, Mitre RM A. Processo Comunicativo e Humanização em saúde. Interface Comun Saúde Educ 2009; 13(Supl. 1):641-649.

40. Gomes AMA, Paiva ES, Valdés MTM, Frota MA, Albuquerque CMA. Fenomenologia, humanização e promoção da saúde: uma proposta de articulação. Saude Soc 2008; 17(1):143-152.

41. Cotta RM M, Cazal M M, Rodrigues JFC, Gomes KO, Junqueira TS. Controle social no Sistema Ú nico de Saúde: subsídios para construção de competências dos conselheiros de saúde. Physis 2010; 20(3):853-872.

Artigo apresentado em 20/06/2011

Aprovado em 10/09/2011

Versão final apresentada em 21/09/2011 